\title{
Effect of control surfaces on the aerodynamic database of the Stratofly hypersonic vehicle
}

\author{
Andrea Flora ${ }^{1,}$, , Pasquale Jr. Capasso ${ }^{1}$, Simona Brancaccio ${ }^{1}$, Paolo Ambrico ${ }^{1}$, Alessio \\ D'Onofrio $^{1}$, Francesco De Stasio ${ }^{1}$ \\ ${ }^{1}$ University of Naples Federico II, Faculty of Aerospace Engineering, Via Claudio, 21, Naples, Italy
}

\begin{abstract}
This paper aims at studying the control surfaces of the STRATOFLY project reference aircraft, funded by the European Commission, under the framework of Horizon 2020 plan. The values of aerodynamic coefficients in a wide range of flow free-stream conditions are stored in the aircraft aerodynamic database. The research goal is to update a pre-existent database that was developed with fixed control surfaces using the six control surfaces deflection as input. Different Mach numbers determine different flow regimes: subsonic, transonic, supersonic, and hypersonic. In subsonic, transonic and low supersonic regimes a vortexlattice solver is used to obtain the global coefficients assuming an unviscous flow on a simplified model. In hypersonic flow a build-up approach is applied: the control surfaces deflection contribution is developed by assuming a two-dimensional flow on the airfoil and by applying shockexpansion theory on the geometry. Then the paper analyses results showing stability and L/D results. The final paragraph focuses on trimmability at cruise Mach. No trimmed solution is obtainable to optimize the propulsive system. The solution proposed to solve this issue is to extend the four elevons: larger elevons are found to be able to trim the vehicle at the desired angle of attack.
\end{abstract}

\section{Introduction}

The STRATOFLY project, funded by the European Commission, under the framework of Horizon 2020 plan, has the aim of assessing the potential of high-speed transport vehicle to reach TRL 6 by 2035, with respect to key technological, societal and economical aspects.

STRATOFLY Academy is an academic challenge launched by this project, this paper is the result of the work of six student of the Academy [1].

Starting from the STRATOFLY MR3 configuration it is important to predict the external aerodynamics of the vehicle in order to conduct a preliminary analysis about flight dynamic and performance. The values of aerodynamic coefficients in a wide range of flow free-stream conditions are needed to carry out this analysis, these values are stored in the Aerodynamic Database of the vehicle (AEDB). A preliminary evaluation of the AEDB is useful to carry out design choices and to have a fast way to evaluate different options. This paper proposes a method to update a given supersonic AEDB developed with fixed control surfaces with the

* Corresponding author: andrea.flora.1996@gmail.com 
effect of the control surfaces' deflection. This method could be applied for any supersonic aircraft, but the results will be reported only for the SRATOFLY MR3 vehicle. A simple analysis of the aircraft with vortex-lattice subsonic and supersonic software has been useful to complete the database and compare the results.

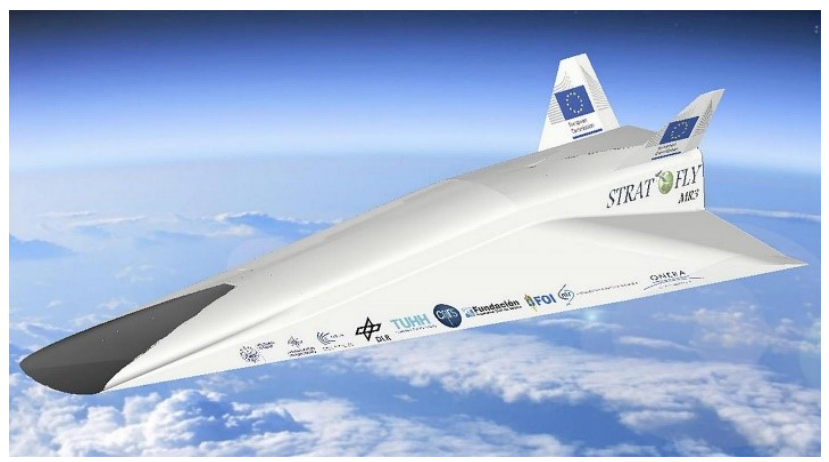

Fig. 1. STRATOFLY vehicle MR3 configuration.

\section{Shock-expansion approach}

In order to study the effect of the control surfaces at high supersonic and hypersonic flows a two-dimensional method has been employed. The motion field has been analysed to obtain the aerodynamic coefficients for checking trim conditions with deflected control surfaces.

\subsection{Matlab code}

The developed code implements shock-expansion theory, that is a combination of oblique shock waves and expansion fans. The pressure ratios on edges of the airfoil have been evaluated thanks to normal shock waves formulas, isentropic and Prandtl-Meyer relations [2]. Then these ratios have been used to obtain aerodynamic coefficients. The bisection method has been utilized to solve numerically shock-expansion relations for evaluating the motion field in each region around the airfoil using undisturbed thermofluid dynamic parameters. Also, when the Prandtl-Mayer angle is larger than 130 degrees the MATLAB code has not been able to evaluate through the bisection method or other root-finding algorithms the pressure jump, that has been considered zero.

The routine is fully parametric so that every airfoil geometry can be imported using six points. It must be specified that all the effects of sideslip and dihedral angles have been considered in this analysis. At first, the estimation of each aerodynamic coefficient has been obtained through well-known resolution around a diamond shaped airfoil. The coefficients depend on Mach number (M) and angle of attack ( $\alpha$ ) [gasdinamica libro]. Then the contribution of the sideslip angle $(\beta)$ has been considered by new definition of the angle of attack $\left(\alpha_{\text {effective }}\right)$ and Mach number for each control surface. Also, the contributions of the dihedral angle and fin's angle of inclination $\left(\mu_{\mathrm{CS}}\right)$ with respect to vehicle's horizontal plane appear in the definition of $\alpha_{\text {effective }}$.

$$
\begin{gathered}
\alpha_{\text {effective }}=\operatorname{tg}^{-1}\left(\frac{\cos (\beta) * \sin (\alpha) * \cos \left(\mu_{\mathrm{CS}}\right)+\sin (\beta) * \cos (\alpha) * \sin \left(\mu_{\mathrm{CS}}\right)}{\cos (\beta) * \cos (\alpha)}\right) \\
\mathrm{M}=\mathrm{M} * \cos (\beta)
\end{gathered}
$$


Multiplicative dimensionless factors have been employed to derive aerodynamic coefficients considering parameters such as characteristic length, wing span and surface.

\subsection{Validation of the two-dimensional approach}

The results obtained with shock-expansion method for different sections of wing have been validated for three different Mach numbers $(M=4,6,8)$ with a comparison with the pressure plots of CFD analyses provided by CIRA [3]. They prove that the hypothesis of twodimensional flow is acceptable, this assumption can be found also in theoretical research [4].

Furthermore, it has been evaluated the ratio between error, that is the difference between pressure obtained from shock-expansion approach and that obtained from CFD analysis, and stagnation pressure at elevon hinge.

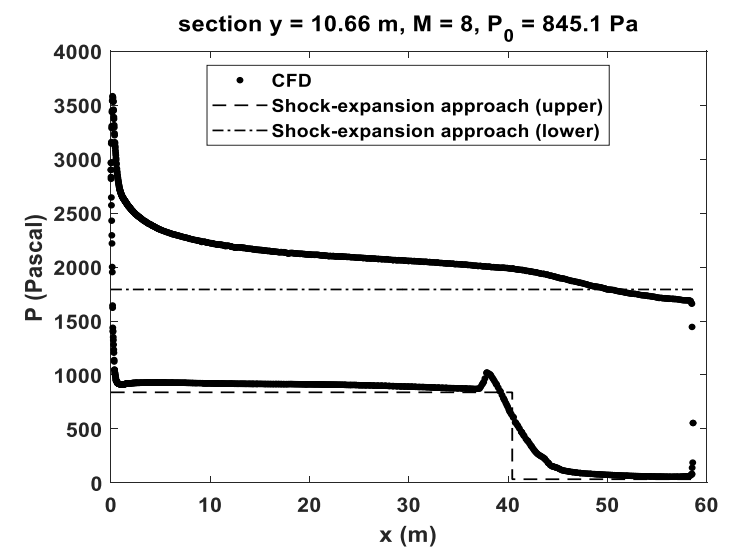

Fig. 2. Comparison between shock-expansion approach and CFD analysis at $\mathrm{M}=8$.

Table 1. Pressure percentage error at elevon hinge.

\begin{tabular}{|c|c|c|}
\hline & Upper & Lower \\
\hline $\mathrm{M}=4$ & $9.9 \%$ & $16 \%$ \\
\hline $\mathrm{M}=6$ & $0.42 \%$ & $17 \%$ \\
\hline $\mathrm{M}=8$ & $3.4 \%$ & $5.8 \%$ \\
\hline
\end{tabular}

\subsection{From sectional to global aerodynamic parameters}

First of all, the aerodynamic coefficients have been estimated for a section. These twodimensional values have been obtained for various sections along the control surfaces (CS) span. Each value has been considered constant from a section to the next one. Finally, the three-dimensional contribution of CS has been provided by integral along the entire CS span.

$$
\Delta C_{I}=\frac{2}{S} \int_{y_{C S_{-} \text {beginning }}}^{y_{C S_{\text {end }}}} C_{i_{\delta} \neq 0}(y) c(y) d y-\frac{2}{S} \int_{y_{C S_{-} \text {beginning }}}^{y_{C S_{\text {_end }}}} C_{i_{\delta}=0}(y) c(y) d y
$$

$\mathrm{I}=\mathrm{Lift}$, drag, side force, roll, pitch, yaw coefficients 
A constant airfoil distribution along the entire CS span has allowed to consider constant the value of aerodynamic coefficient and equal to its value at the centre of CS span. This approach has been used to calculate all aerodynamic contributions [5].

$$
\begin{gathered}
\Delta \mathrm{C}_{\mathrm{I}}=\frac{2}{\mathrm{~S}} \mathrm{C}_{\mathrm{i}_{\delta \neq 0}}\left(\mathrm{y}=\mathrm{y}_{\mathrm{CS} \text { centerline }}\right) \int_{\mathrm{y}_{c s_{\text {eneginning }}}}^{\mathrm{y}_{c s}} \mathrm{c}(\mathrm{y}) \mathrm{dy}-\frac{2}{\mathrm{~S}} \mathrm{C}_{\mathrm{i}_{\delta=0}}(\mathrm{y}= \\
\left.\mathrm{y}_{C \mathrm{CS}_{\text {centerline }}}\right) \int_{\mathrm{y}_{c s_{\text {beginning }}}}^{\mathrm{y}_{c s}} \mathrm{c}(\mathrm{y}) \mathrm{dy}
\end{gathered}
$$

\section{Database assembly}

This paper proposes a simple method to update a given aerodynamic database of a supersonic vehicle, in this case the Stratofly MR3 reference vehicle, with the effect of the control surfaces deflection.

The generic aerodynamic coefficient $C_{I}$ has been given in the original AEDB as a tabular function of $M, \alpha$ and $\beta$ in a given range of values of these parameters.

To evaluate the contribution of the control surfaces the following approach has been used.

Fixing M, $\alpha$ and $\beta$, the contribution to the generic "I" coefficient of the surfaces' angular deflection, calculated as already shown, has been added to the initial value:

$$
\mathrm{C}_{\mathrm{I}, \text { fixed }}(\mathrm{M}, \alpha, \beta)+\sum_{\mathrm{k}} \Delta \mathrm{C}_{\mathrm{I}, \text { single surface } \mathrm{k}}\left(\mathrm{M}, \alpha, \beta, \delta_{\mathrm{k}}\right)
$$

Where $\delta_{\mathrm{k}}$ is the angular surface deflection of the kth control surface. This has been replicated for all the control surfaces, considering the sign of the contribution with respect to the deflection convention, the kind of coefficient and the specific control surface.

For example, in this application, the contribution to a symmetric coefficient (e.g. CL, CM...) of the right and left elevons is equal.

$$
\Delta \mathrm{C}_{\mathrm{I}, \text { right elevon }}=\Delta \mathrm{C}_{\mathrm{I}, \text { left elevon }}
$$

Instead the contribution to an antisymmetric coefficient (e.g. $\mathrm{Cl}, \mathrm{Cn} . .$. ) of the right and left elevons is equal and opposite.

Repeating this procedure for all desired values of deflections (or even all possible combinations of deflections) is possible to store the updated database.

$$
\mathrm{C}_{\mathrm{I}}\left(\mathrm{M}, \alpha, \beta, \delta_{\mathrm{el}}, \delta_{\text {rud }}\right)=\mathrm{C}_{\mathrm{I}, \mathrm{fixed}}(\mathrm{M}, \alpha, \beta)+\Delta \mathrm{C}_{\mathrm{I}, \mathrm{el}}\left(\mathrm{M}, \alpha, \beta, \delta_{\text {el }}\right)+\Delta \mathrm{C}_{\mathrm{I}, \mathrm{rud}}\left(\mathrm{M}, \alpha, \beta, \delta_{\text {rud }}\right)
$$

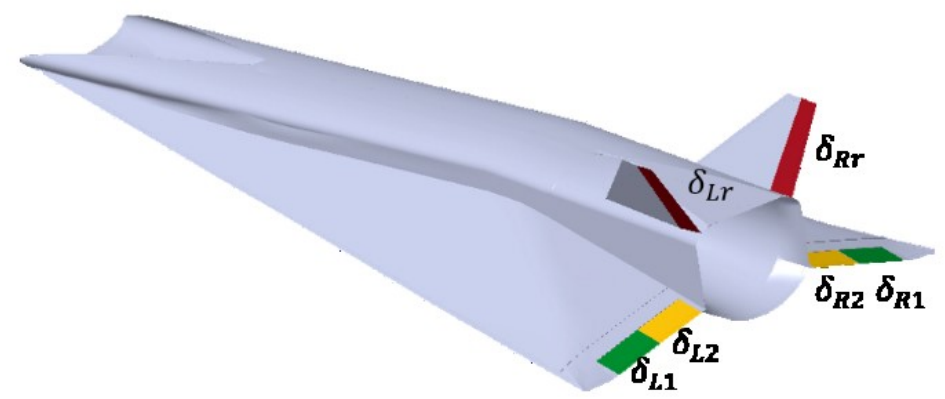

Fig. 3. Convention of control surfaces of the reference vehicle. 


\section{Results}

An overview of the most significant results follows. This method has been able to evaluate the contribution of the control surface and all the trends and shifts resulting from deflection are what the supersonic literature predicts referred to similar case studies.

All moment coefficients has been calculated referring to the maximum forward position of centre of gravity (X_CoG $=-49.98 \mathrm{~m}$ about XYZ reference frame). To maximize the effect of the control surfaces, they have been deflected simultaneously.

The negative slope of pitching moment coefficient shows longitudinal stability. The positive deflection of the elevons (downwards) shifts up the lifting coefficient plot and down the pitching moment coefficient while the negative deflection has the opposite effect. The positive slope of yawing moment coefficient shows lateral stability.

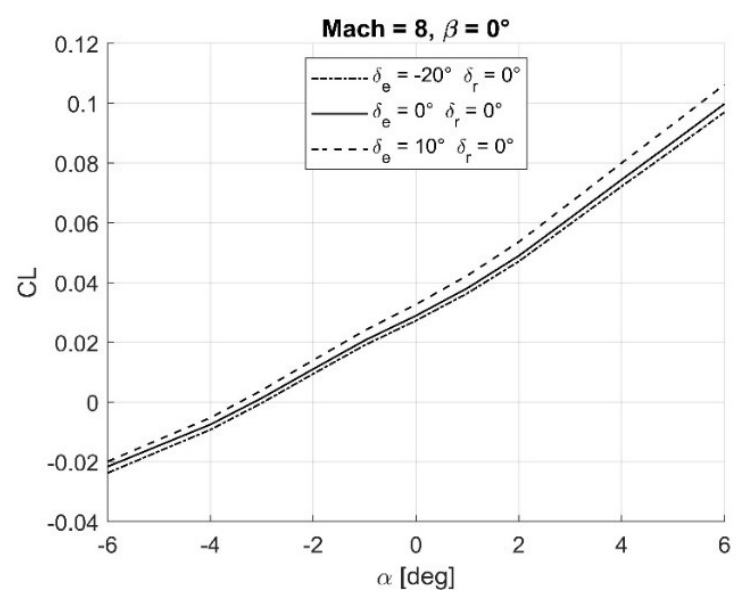

Fig. 4. Effect of elevons' deflection on lifting coefficient vs $\alpha$ at $M=8$.

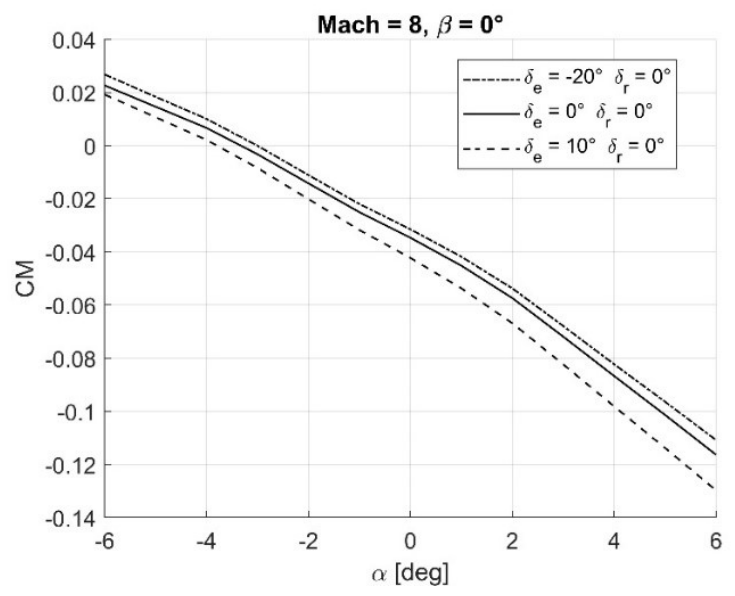

Fig. 5. Effect of elevons' deflection on pitching moment coefficient vs $\alpha$ at $M=8$. 


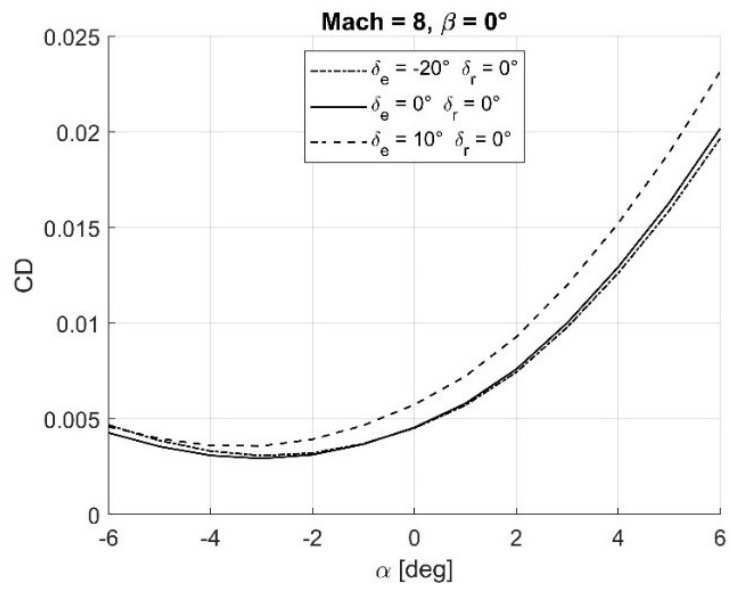

Fig.6. Effect of elevons' deflection on drag coefficient vs $\alpha$ at $\mathrm{M}=8$.

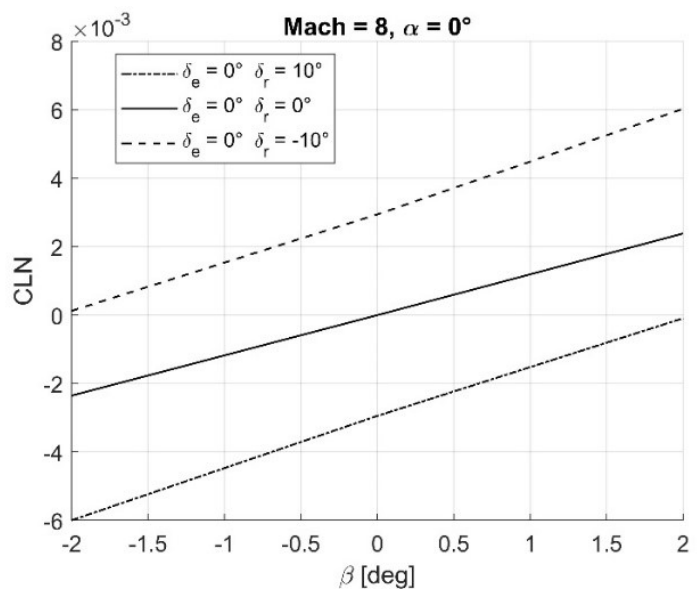

Fig. 7. Effect of rudders' deflection on yawing moment coefficient vs $\beta$ at $M=8$.

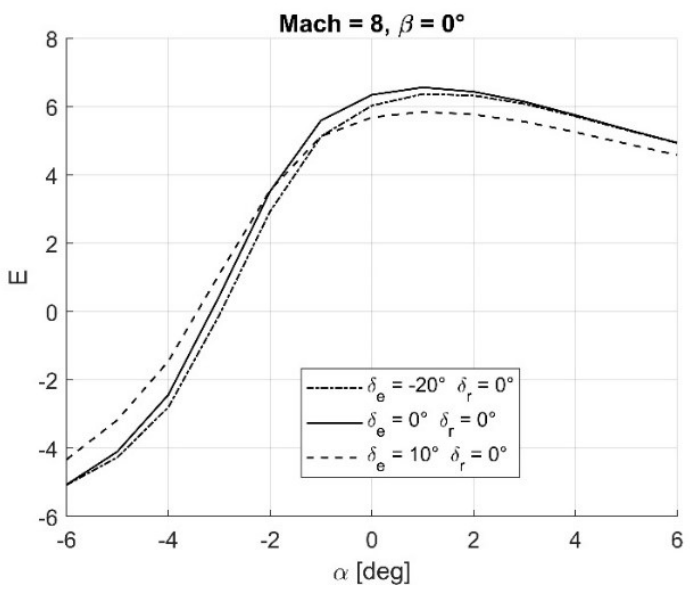

Fig. 8. Effect of elevons' deflection on efficiency vs $\alpha$ at Mach $=8$. 


\section{Trim analysis}

One design goal is to have a trimmed condition compatible with the maximum efficiency of the propulsion system. Analyzing the trimmability of the vehicle in high supersonic and hypersonic flow regimes, at Mach of cruise $(M=8)$, the trim angle of attack is between $-3,8^{\circ}$ and $-3^{\circ}$ while the maximum intake efficiency angle of attack is equal to $-2^{\circ}$.

In order to obtain the required trim condition an expansion of the elevons has been applied. Through the shock-expansion method proposed, the contribution of $6 \mathrm{~m}$ longer elevons has been evaluated. This length increment has been found by iterating the method until the trim angle has been optimized.

Negative deflections of the elevons allow to achieve the optimal trim condition but, at the same time, have a smaller effect on pitching moment coefficient than the positive ones.
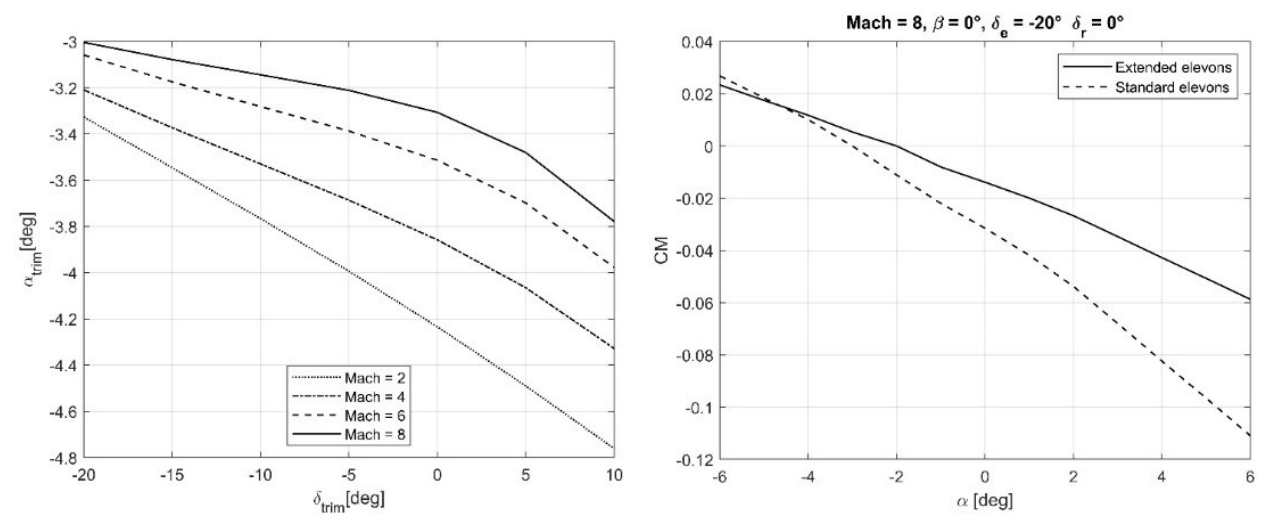

Fig. 9. Angle of trim for different Mach numbers in function of elevons' deflection and pitch coefficient with extended and standard elevons.

\section{Vortex Lattice software}

In order to carry out the subsonic and low supersonic regimes analysis of the effect of the control surfaces deflections, two software have been used. Specifically, AVL [6] for subsonic regime and OpenVSP for both subsonic and low supersonic regimes. They are based on Vortex lattice method and inviscid linearized potential flow theory, which are historically known to be a very useful preliminary design tool [7].

\subsection{Vortex Lattice method theoretical background}

Vortex Lattice is a numerical method that calculates lift curve slope, induced drag and lift distribution for a given aircraft configuration. The wing is modeled with horseshoe vortices distributed along span and chord. Effects of both thickness and viscosity are neglected by Biot-Savart Law, Kutta-Joukovsky theorem, Hermann von Helmhotz theory, Prandtl liftingline theory [8]. While at first supersonic applications of Vortex Lattice method were ignored, then there was an extension of vortex lattice techniques to supersonic Mach numbers [7]. 


\subsection{Obtaining the geometry}

Each software has its interface to recreate the basic shape of the aircraft of interest. OpenVSP in particular allows the creation of a three-dimensional model through common engineering parameters, while for AVL an input file has to be written according to software requirements.

The final result is a wave-rider configuration, with no fuselage. It has the wing with four elevons, two internal elevens and two external ones, and the fins; on each of them a two rudder is placed, for a total of two. It has been possible to import the actual airfoil shapes for wing and the fins.

The geometry files of both software have been created with a series of intermediate models though a trial-and-error approach. The main problem which has surfaced is the modelling of the intake. When analysing the model that mostly resembles the MR3, both software have crashed due to the inlet's complex geometry. A compromise between resemblance and simplicity has been reached.

\section{Conclusions}

In conclusion, this approach can be used to evaluate the effect of the control surfaces on a given aerodynamic database. This paper shows a single application of the method and its possible utility in a preliminary design phase. The routine can be applied also on other aircrafts, and it can be fully validated with experimental tests [9].

The objective of the STRATOFLY academy challenge has been fulfilled. A complete aerodynamic database of the reference vehicle has been produced combining the vortex lattice results with the proposed shock-expansion method.

\section{References}

1. N. Viola, R. Fusaro, B. Saracoglu, C. Schram, V. Grewe, M. Marini, R. Scigliano, S. Hernandez, D. Hauglustaine and C. Fureby, STRATOFLY academy: inspire young generations and be inspired by new ideas (2019).

2. G. M. Carlomagno, Elementi di Gasdinamica (2009).

3. R. Scigliano, M. Marini, P. Roncioni, R. Fusaro, N. Viola, STRATOFLY high-speed propelled vehicle preliminary aerothermal design (2019).

4. http://dma.ing.uniroma1.it/users/ls_gas/MATERIALE/cap7.pdf.

5. J. Roskam, Airplane Flight Dynamics \& Automatic Flight Controls (1995).

6. K. Budziak, Aerodynamic Analysis with Athena Vortex Lattice (2015).

7. L. R. Miranda, R. D. Elliott, W. M. Baker, A generalized Vortex Lattice method for subsonic and supersonic flow applications (1977).

8. John D. Anderson Jr., Fundamentals of Aerodynamics (1984).

9. T. Bykerk, D. Verstraete, S. Wolf, V. F. Villace, J. Steelant, Performance and stability analysis of a hypersonic vehicle for a low speed flight test program (2018). 